\title{
Evaluation of a brief pilot nutrition and exercise intervention for the prevention of weight gain in general practice patients
}

\author{
Alison O Booth ${ }^{1, *}$, Caryl A Nowson ${ }^{1}$, Nancy Huang ${ }^{2}$, Catherine Lombard ${ }^{2}$ \\ and Kate L Singleton ${ }^{2}$ \\ 'The Centre for Physical Activity and Nutrition, School of Exercise and Nutrition Sciences, Deakin University, \\ 221 Burwood Highway, Burwood, Victoria 3125, Australia: ${ }^{2}$ The Victorian Council on Fitness and General \\ Health (VICFIT), Collins Street, Melbourne, Victoria, Australia
}

Submitted 24 August 2005: Accepted 31 January 2006

\begin{abstract}
Objective: To pilot-test a brief written prescription recommending lifestyle changes delivered by general practitioners (GPs) to their patients.

Design: The Active Nutrition Script (ANS) included five nutrition messages and personalised exercise advice for a healthy lifestyle and/or the prevention of weight gain. GPs were asked to administer 10 scripts over 4 weeks to 10 adult patients with a body mass index (BMI) of between 23 and $30 \mathrm{~kg} \mathrm{~m}^{-2}$. Information recorded on the script consisted of patients' weight, height, waist circumference, gender and date of birth, type and frequency of physical activity prescribed, and the selected nutrition messages. GPs also recorded reasons for administering the script. Interviews recorded GPs views on using the script.

Setting: General practices located across greater Melbourne.

Subjects and results: Nineteen GPs (63\% female) provided a median of nine scripts over 4 weeks. Scripts were administered to 145 patients (mean age: $54 \pm 13.2$ years, mean BMI: $31.7 \pm 6.3 \mathrm{~kg} \mathrm{~m}^{-2} ; 57 \%$ female), $52 \%$ of whom were classified as obese (BMI $>30 \mathrm{~kg} \mathrm{~m}^{-2}$ ). GPs cited 'weight reduction' as a reason for writing the script for $78 \%$ of patients. All interviewed GPs $(90 \%, n=17)$ indicated that the messages were clear and simple to deliver.

Conclusions: GPs found the ANS provided clear nutrition messages that were simple to deliver. However, GPs administered the script to obese patients for weight loss rather than to prevent weight gain among the target group. This has important implications for future health promotion interventions designed for general practice.
\end{abstract}

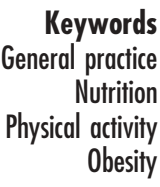

The prevalence of obesity in the Australian population rose from $9 \%$ in $1989 / 90$ to $15 \%$ in $2001^{1}$. Sixty-five per cent of male and $45 \%$ of female adults were overweight or obese in $2001^{2}$. Not only does obesity contribute to an estimated 5000 deaths each year in Australia, it also poses a major risk factor for chronic diseases including cardiovascular disease, stroke, type II diabetes mellitus, atherosclerosis, hypertension, gallbladder disease and some cancers $^{3,4}$. Many of these diseases can often be treated and prevented through improvements in nutrition and/or increasing physical activity. Considering that general practitioners (GPs) in Australia have access to over 90\% of the population in the course of a year ${ }^{5}$ and are viewed by the general public as being reliable and credible sources of nutrition information ${ }^{6}$, general practice is likely to be an ideal setting for health promotion.

Approximately $50 \%$ of visits to GPs are made by overweight or obese patients ${ }^{7}$ and approximately $25 \%$ of all visits are nutrition-related ${ }^{8}$. Consequently, GPs have many opportunities to offer nutrition advice. Studies have demonstrated that GPs can be effective in improving health behaviours such as increasing smoking cessation rates $^{9,10}$, reducing alcohol consumption ${ }^{11}$ and increasing physical activity ${ }^{12,13}$. One study suggested that patients may be more likely to lose weight if prompted by their $\mathrm{GP}^{14}$. Nutrition advice has also proved to be effective when offered as part of a time-intensive intervention ${ }^{15}$. However, as time is a major limitation for practitioners, nutrition/lifestyle information needs to be available in a condensed form that can be provided to patients during a standard consultation.

The Active Script Programme (ASP) is a brief and effective physical activity intervention used in general practice. Developed in 1999, the ASP is run by the Victorian Council on Fitness and General Health (VICFIT). The aim of this intervention is to increase the number of GPs who deliver brief, effective physical activity advice to inactive patients ${ }^{16}$. GP outcomes attributed to the ASP include improved competence in promoting physical activity; increased knowledge of the benefits of physical 
activity; and improvements in assessing, managing and counselling patients in relation to physical activity. The ASP has also resulted in an increase in knowledge and physical activity levels among patients ${ }^{16}$. However, VICFIT recognised that more effective lifestyle advice should include both nutrition and physical activity recommendations. VICFIT set up an advisory group who then developed specific nutrition advice to complement the physical activity advice in the ASP. This led to the development of the Active Nutrition Prescription (ANS), a brief $(<5 \mathrm{~min})$ nutrition and physical activity tool for use in general practice. The aim of the present study was to evaluate GPs' use of the ANS in general practice to prevent weight gain among the healthy to overweight population.

\section{Methods}

\section{Development of the ANS}

VICFIT convened a small expert working party with representatives from education, government and nongovernment organisations to provide content expertise in the development of the ANS. Over several meetings, the working party identified overall themes to be explored and finally developed five specific nutrition messages based on the most recent scientific evidence for the prevention of weight gain. The ANS was developed in the form of a paper-based script pad with a view to create an electronic version after the pilot study. Once the ANS script pad was developed and the target group was defined, input into further development of the tool was gathered from GPs, consumers and key stakeholders before the tool was pilot-tested in general practices.

\section{Target group}

The ANS was targeted at people with a body mass index (BMI) of between 23 and $30 \mathrm{~kg} \mathrm{~m}^{-2}$, and was aimed at preventing weight gain and improving nutritional habits among this group. The ANS was not designed to result in weight loss in the short term, but had the potential to prevent weight gain in the long term. Therefore, it is not an appropriate treatment for obesity where weight loss is the goal. The working party recognised that the obese group would require more intensive treatment.

\section{Nutrition messages}

The nutrition messages developed were:

1. Eat five servings of vegetables each day;

2. Eat two servings of fruit each day;

3. Drink water in place of sweetened soft drinks, cordial, sports drinks and fruit juice;

4. Limit high-fat convenience and take-away foods to once per week; and

5. If hungry, snack on fruit and vegetables between meals.

\section{Script pad (ANS)}

The ANS script pad included three sections: patient details, a physical activity component and a nutrition component. In the first section, the GP documented the patient's date of birth, sex, height, weight, and waist circumference. The second section was for physical activity prescription and includes type, levels and frequency of activity recommended. In the third section, the GP has the option to select any combination of the five nutrition messages they think are applicable to the patient. Each script could be personalised, with additional space for comments, an option to refer the patient to a dietitian, and a space to indicate a time for a review or to make a follow-up appointment. There was additional information that included examples of serving sizes, alternative snack options and other general nutrition advice.

\section{GP pilot test}

\section{Recruitment and education}

Some GPs were recruited by personal invitation at GP conferences and seminars; however, many were recruited via a divisional newsletter. Victorian Divisions were contacted and asked to place an advert in their regular newsletter requesting expressions of interest from GPs to participate in the pilot study of the ANS. Interested GPs who faxed back a response were sent a letter of invitation. Recruited GPs were asked to administer the prescription to 10 suitable patients over a 2 -week period if full-time or a 4-week period if part-time, but were given more time if needed. Participating GPs were visited at their practices by an ANS representative and given a brief (approximately $15 \mathrm{~min}$ ) education session in which they were shown how to use the script, given details about the target group and given the script pad. The script pad included 10 carbon copies that were collected for analysis post-intervention. A semi-structured telephone interview was administered by a research assistant within 7 days of the completion of the scripts. This phone interview lasted approximately $15 \mathrm{~min}$ and each GP was reimbursed \$AUD 50 for his or her time.

\section{Feedback form regarding patients}

Each GP was asked to fill in a feedback form after writing each script. The GP was asked to note the initial reason for the visit, diagnosis, additional comments, and the reason for writing the script. The latter was indicated by ticking one or more options, consisting of weight reduction, weight maintenance, poor nutrition knowledge, poor nutrition/ activity habits, patient requested the advice, patient not active, chronic disease or to motivate the patient.

\section{Materials provided to the GP}

Each GP was supplied with one script pad of 10 scripts with carbon copies, 10 feedback forms (regarding patients), a GP information sheet, and six to 10 physical activity patient questionnaires, which included a physical 
activity scoring sheet. The physical activity patient questionnaire and scoring sheet were used to briefly assess physical activity level. These physical activity patient questionnaires were provided but were not compulsory to complete (data not collected). The GP information sheet included information on the target group; evidence supporting the nutrition messages; a list of resources; details on physical activity assessment; how to measure waist circumference and BMI; and risk of disease at different levels of waist circumference and BMI categories. GPs were also provided with 10 orange cards with details of VICFIT's Physical Activity Infoline number to be given to recipients of the script at the GP's discretion.

\section{Results}

\section{GP characteristics}

Of the 32 GPs who expressed interest in participating in the pilot study, 25 were trained in how to apply the ANS and 19 completed or partially completed the required number of scripts (63\% female). Reasons given by the GPs for not participating once trained included a lack of time, being away on holidays, forgetting to participate and believing that participation in the study was too difficult.

\section{Patient characteristics}

Active Nutrition Scripts were written for 145 patients, with a median of nine scripts written per GP. Of the patients whose gender was recorded (83\%), 69\% were female. The mean ( \pm standard deviation) waist circumference of the patients was $105.9 \pm 11.9 \mathrm{~cm}$ for males and $98.4 \pm 15.5 \mathrm{~cm}$ for females. Mean BMI was in the obese range (Table 1). Fortythree per cent of patients were in the target group (i.e. BMI of between 23 and $30 \mathrm{~kg} \mathrm{~m}^{-2}$ ) and 52\% were obese.

\section{Physical activity prescription}

GPs prescribed physical activity to $97 \%$ of ANS recipients. GPs prescribed walking to the majority of patients (61\%), with gym exercises being the next most frequently prescribed activity (4\%) for the recipients. Twenty-five per cent received exercise prescription without a suggestion for a specific activity. Thirty minutes of daily activity was prescribed to 69\% of patients. GPs prescribed $65 \%$ of patients to exercise 5-7 times per week, $19 \%$ to

Table 1 Patient characteristics

\begin{tabular}{lccc}
\hline & & \multicolumn{2}{c}{ Mean \pm SD } \\
\cline { 3 - 4 } & Range & Males & Females \\
\hline Age (years) & $19-86$ & $54.7 \pm 12.7$ & $53.8 \pm 13.9$ \\
Height $(\mathrm{cm})$ & $135-191$ & $172.7 \pm 10.1$ & $161.1 \pm 6.8$ \\
Weight (kg) & $44-134$ & $93.3 \pm 17.1$ & $81.8 \pm 18.3$ \\
BMl (kg m${ }^{-2}$ ) & $18.7-50.1$ & $31.5 \pm 5.2$ & $31.6 \pm 6.8$ \\
Waist circumference $(\mathrm{cm})$ & $68-150$ & $105.9 \pm 11.9$ & $98.4 \pm 15.5$
\end{tabular}

SD - standard deviation; BMI - body mass index. exercise $4-5$ times per week and $8 \%$ to exercise $1-2$ times per week (data were missing for $8 \%$ of the prescriptions).

\section{Nutrition prescription}

GPs ticked all five nutrition boxes for $55 \%$ of recipients. GPs did not offer nutrition advice for $3 \%$ of the recipients (i.e. these recipients only received physical activity prescription). More than $80 \%$ of all patients received advice to consume two servings of fruit each day, five servings of vegetables each day and to drink water in place of sweet drinks (Table 2). GPs ticked the box 'drink water in place of sweetened soft drinks, cordial, sports drinks and fruit juice' more frequently for overweight/obese (BMI $>25 \mathrm{~kg} \mathrm{~m}^{-2}$ ) patients than they did for patients in the healthy weight range (BMI $\leq 25 \mathrm{~kg} \mathrm{~m}^{-2}$ ) (Table 3). GPs ticked the water and take-away messages more frequently for patients under the age of 50 years than they did for those over the age of 50 (Table 3).

\section{Referral to a dietitian/review/full assessment}

Sixteen patients (11\%) were referred to a dietitian, 106 (73\%) were asked to make a follow-up appointment (review) and 17 (12\%) were asked to make an appointment for a full dietary, physical activity and social assessment with the GP so that a more thorough weight management plan could be developed at a future date. Of the $73 \%$ of patients who received a recommendation for a review, the median months for the review was 2 months for males and 1 month for females. The patients who did not receive a recommendation for a review were less likely to be referred to a dietitian $(P=0.048)$, less likely to be asked to make an appointment for a full assessment $(P=0.006)$, were older $(P=0.019)$, had a lower BMI $(P=0.005)$ and were less likely to be asked to increase their vegetable intake to at least five servings per day $(P=0.009)$.

\section{Feedback form - reported reasons for GP visit, diagnoses and administration of the script}

Patient information was available for $95 \%$ of patients who received an ANS prescription. The main reasons for the GP visit, as recorded by the GP, included a blood pressure check (22\%), a general check-up (18\%), a session addressing weight concerns (16\%), obtaining a repeat script (10\%) and a cholesterol check (9\%). The diagnoses included overweight (43\%), hypertension (26\%), lipid disorders (19\%), diabetes (11\%) and others (9\%). Encouraging weight reduction was the main reason given by GPs for writing the script (78\%), followed by efforts to motivate the patient (48\%), reduce inactivity (30\%), address poor nutrition or activity habits (23\%) and reduce chronic disease (19\%).

\section{GP interview}

Seventeen of the 19 participating GPs were interviewed (90\%) following completion of the scripts. The mean number of years in practice was 23 (range: 3-40 years), 
Table 2 Percentage each nutrition advice/message box was ticked in relation to gender

\begin{tabular}{|c|c|c|c|}
\hline \multirow[b]{2}{*}{ Nutrition advice } & \multicolumn{3}{|c|}{$\%$ ticked } \\
\hline & Males $(n=37)^{*}$ & Females $(n=83)^{*}$ & Total $(n=145)$ \\
\hline Eat five servings of vegetables each day & 84 & 82 & 83 \\
\hline Eat two servings of fruit each day & 84 & 80 & 81 \\
\hline Drink water in place of sweetened soft drinks, cordial, sports drinks and fruit juice & 81 & 77 & 81 \\
\hline Limit high-fat convenience and take-away foods to once per week & 73 & 70 & 74 \\
\hline If hungry, snack on fruit and vegetables between meals & 81 & 76 & 77 \\
\hline
\end{tabular}

* Gender was recorded in $83 \%$ of patients.

with a mean of 99 patients seen each week (range: 20-200). Ten GPs worked part-time. The time reported for delivering the script was approximately 4.9 min per script. All interviewed GPs indicated that the messages were clear and simple to deliver, and would have liked to continue using the script post-pilot. Forty-seven per cent stated that they would be more likely to initiate a nutrition or physical activity discussion with their patients in the future and 29\% reported that they were now more likely to routinely ask new patients about nutrition and physical activity.

\section{Discussion}

This study explored GPs' use of a brief nutrition and exercise prescription (ANS) that was developed to complement an existing exercise prescription intervention for a more integrated lifestyle resource for GPs. Although GPs were instructed to administer the ANS to the healthy and/or overweight population, more than half of the patients who received the ANS were obese. However, the obese population is likely to require more intensive interventions and the ANS alone is unlikely to be effective in reducing weight among this group.

There are a number of possible reasons why GPs administered the ANS to obese patients rather than the target group consisting of healthy and/or overweight patients. First, GPs may be keener to use the ANS with the obese population as this group may be viewed to be in greater need of an intervention than healthy/overweight persons. This supports findings that GPs prefer to use nutrition intervention for treatment rather than health promotion $^{17,18}$. GPs choosing to intervene more often for obese patients than for overweight patients has occurred previously $^{18-20}$.

Second, the increasing percentage of obese and overweight persons in the population may make it difficult to distinguish overweight people from obese people. Low levels of obesity identification have occurred in previous studies $^{18,21-24}$. GPs may find it difficult to distinguish between those who are overweight and those who are obese, if BMI is not calculated. GPs were asked to record height and weight on the ANS, and were recommended to calculate BMI. As there were no data to indicate whether BMI was calculated, it could be that GPs were unaware of the BMI of their patients and made an assessment of their BMI visually.

Third, GPs may have seen a need to assist their obese patients with weight management but had few options available to them and, thus, perceived the ANS as an appropriate substitute.

There is a need for nutrition interventions to be brief; however, they also need to be effective for patients. Practitioners in The Eating Patterns Study provided a selfhelp nutrition education booklet along with a motivational message to patients in less than $3 \mathrm{~min}^{25}$. Although both the control and intervention groups reduced their fat intake, the changes were significantly larger for the intervention group at the 3- and 12-month follow-ups. Another brief nutrition education tool, the Patient Information Letters (PIL) developed by the Dutch College of General Practitioners $^{26}$, provided nutrition advice on selected diagnoses. Only 5\% of physicians reported using the letters sufficiently, with $63 \%$ agreeing that they did not use them enough. The main barriers to using the PIL included not thinking of it at the right moment, not knowing the content well enough and finding it too time-consuming ${ }^{26}$.

Although some GPs feel that they can be effective in nutrition intervention and agree that nutrition is important in managing disease ${ }^{27}$, they generally lack confidence in offering more detailed nutrition advice due to insufficient knowledge, which is a result of gaps in nutrition training throughout undergraduate and postgraduate studies. Other barriers to offering nutrition advice include time constraints, a lack of incentives or reimbursements, inadequate support materials, complex advice to offer, lack of training in counselling skills, and a long delay between intervention and observable effects ${ }^{26,28}$. Where interventions were designed specifically for weight reduction, some studies have revealed that GPs felt their influence on weight reduction was limited compared with other professionals ${ }^{27,29}$. However, other studies have found that GPs rated themselves as 'quite effective' in influencing patients' $\operatorname{diets}^{30}$ and influencing patients to lose weight ${ }^{19}$.

Many studies designed for weight-loss purposes are often more time-intensive than the current study and have included detailed dietary assessments, weight management plans ${ }^{31}$, multiple GP training sessions and frequent patient visits ${ }^{15,18}$. For example, the Counterweight 
Programme in the $\mathrm{UK}^{15,18}$ included lifestyle advice offered either individually or as part of a group on frequent occasions (six 10-30 min appointments over 3 months for the individual sessions or six $1 \mathrm{~h}$ group sessions), with quarterly follow-ups recommended. In the Counterweight Programme, $34 \%$ of patients lost $5 \%$ or more of their initial weight in 12 months. Although the programme was effective, it was time-intensive and may be difficult to apply permanently within a general practice setting.

Cost-effective health promotion delivery methods during consultation might include the use of other allied health personnel. For example, it may be more reasonable for practice nurses (PNs) to run such health promotion programmes for population health outcomes, particularly to minimise any time concerns of the GP. On average, GP consultation time is $14.6 \mathrm{~min}$ per patient (median $12 \mathrm{~min})^{32}$, which does not allow much room for lifestyle intervention. In the Counterweight Programme, only 15\% of GPs reported spending up to $10 \mathrm{~min}$ of the consultation discussing weight, compared with $76 \%$ of $\mathrm{PNs}^{15,18}$. A survey conducted by Steptoe et al. ${ }^{33}$ on 107 GPs and 58 PNs in the UK revealed the majority of GPs and PNs agreed that it was most appropriate for PNs to deliver health promotion advice to their patients. The majority of PNs also stated they had the time for preventive medicine, as opposed to only 30\% of GPs. Although utilising PNs for health promotion in general practice would remove some of the time constraints placed on GPs, few studies have assessed patient attitudes and consequent behaviour change towards receiving advice from a $\mathrm{PN}$ as opposed to a GP. Moreover, less than half of all practices in Australia employ PNs or health professionals other than GPs.

Along with barriers that can be overcome, such as a lack of knowledge and limited available resources ${ }^{34,35}$, other barriers including time constraints and a lack of reimbursement may limit GPs' ability to offer detailed lifestyle assessments and provide individualised weight management plans on a frequent basis. If time constraints cannot be easily altered and increasing reimbursement to GPs is found to be too expensive, it may be more realistic that lifestyle advice is provided by nurses or allied health personnel. GP practice has traditionally been treatment-focused, which is likely to be a further barrier preventing GPs from participating in health promotion activities.

Less time-intensive interventions such as the ANS are likely to be adopted more readily by GPs due to their simplicity and usability within a usual consultation; however, the effectiveness of such brief interventions in general practice needs to be evaluated further. The ANS pilot study assessed the acceptability of the ANS for GPS. No information was collected on the effectiveness of the ANS for the patients in terms of increasing physical activity and/or improving eating behaviours, and this must be assessed before this programme can be implemented on a population level. 


\section{Limitations}

One of the limitations of this study was the limited number of GP participants. Participating GPs were more likely to be interested in nutrition than the general GP population. There was also a high rate of incomplete or incorrectly filled out scripts. For example, some patients received exercise prescription without any specific activity to partake in. Gender and waist circumference data were missing for $17 \%$ and $23 \%$ of patients, respectively. Finally, GPs generally took 3-6 weeks to write nine scripts. This figure has the potential to be higher considering that about half of all GP visits are made by overweight or obese persons ${ }^{7}$ and the GPs in this study saw about 100 patients per week. However, the newness of the ANS and the brief training may have contributed to this low number, along with the traditional barriers towards utilising health promotion in the general practice setting.

\section{Conclusions and recommendations}

GPs found the ANS messages and process to be acceptable in the clinical setting. GPs administered the script to obese patients for the purpose of weight loss despite being instructed to administer the script to healthy and overweight patients to prevent weight gain. GPs may not have been aware of who was obese as BMI was not necessarily recorded and documented. In addition, GPs may have been resistant to initiate preventive health messages as their traditional role is related to treatment delivery. Future research needs to identify barriers to GP attitudes and behaviour towards using health promotion interventions with lower-risk groups. Additionally, an assessment is needed on whether other health practitioners can provide effective lifestyle advice, with the support of GPs, resulting in patient behaviour change.

Increasing awareness and identification of obesity should be a priority for public health policy. An option includes advising GPs to calculate BMI for all patients to assist with overweight and obesity identification. Education on the benefits of health promotion should also be implemented. To improve BMI identification for the ANS, BMI could be calculated by the GP and recorded on the script. Utilising other allied health staff, where possible, could alleviate some time constraints. Referral options for obese patients also need to be identified. Finally, tools such as the ANS need to be integrated into the practice systems and processes to improve uptake. Process barriers within the practice need to be assessed by identifying support available and resources needed.

If the ANS was to be implemented more widely, it would be important to develop a method that could determine the effect of the ANS at the patient level and to gain patients' input and opinions towards receiving the ANS. The GP education session may also need to be revised to educate and support GPs in using the ANS on the target population and to further educate GPs in correctly and completely filling in the script.

Preventing weight gain is important in tackling the obesity epidemic that faces the world today. Nations, particularly Australia, the UK and the USA, are becoming more and more overweight. The average weight has increased significantly over the past decade; therefore, an intervention designed to prevent weight gain is clearly beneficial. A brief tool such as the ANS has the potential to contribute towards this goal as it can be implemented by GPs within a normal consultation time.

\section{Acknowledgements}

The researchers gratefully acknowledge the contribution of Ms Veronica Graham, Public Health Nutritionist for the Department of Human Services Victoria; the Bendigo \& District Division of General Practice consumer group and staff; and participating GPs. The following key stakeholders are also acknowledged: Dietitians Association Australia, Diabetes Australia, National Heart Foundation, SIGNAL, Nutrition Australia, General Practice Divisions Victoria, The Commonwealth Department of Health and Ageing, Department of Human Services Victoria, International Diabetes Institute, The Cancer Council Victoria, Jean Hailes Foundation, Professor Boyd Swinburn and Associate Professor Gary Eggar. This work was supported by the Commonwealth Department of Health and Ageing and VICFIT Incorporated.

\section{References}

1 Australian Bureau of Statistics (ABS). National Health Survey. Report No. 4364.0. Canberra: ABS, 2001.

2 Cameron AJ, Welborn TA, Zimmet PZ, Dunstan DW, Owen $\mathrm{N}$, Salmon J, et al. Overweight and obesity in Australia: The 1999-2000 Australian Diabetes, Obesity and Lifestyle Study (Ausdiab). Medical Journal of Australia 2003; 178(9): 427-32.

3 World Health Organization (WHO). Obesity and Overweight. Geneva: WHO, 2003.

4 Bray GA. Medical consequences of obesity. Journal of Clinical Endocrinology and Metabolism 2004; 89(6): 2583-9.

5 Commonwealth Department of Health and Aged Care. General Practice in Australia: 2000. Canberra: Commonwealth of Australia, 2000.

6 Serra-Majem LL, Calvo JR, Male ML, Ribas L, Lainez P. Population attitudes towards changing dietary habits and reliance on general practitioners in Spain. European Journal of Clinical Nutrition 1999; 53(Suppl. 2): S58-61.

7 Britt H, Miller G, Knox S, Charles J, Valenti L, Pan Y, et al. General Practice Activity in Australia 2003-04. Sydney: Australian Institute of Health and Welfare and the University of Sydney, December 2004.

8 Kolasa KM. Developments and challenges in family practice nutrition education for residents and practicing physicians: an overview of the North American experience. European Journal of Clinical Nutrition 1999; 53(Suppl. 2): S89-96.

9 Wilson DH, Wakefield MA, Steven ID, Rohrsheim RA, Esterman AJ, Graham NM. 'Sick of smoking': evaluation of a targeted minimal smoking intervention in general practice. Medical Journal of Australia 1990; 152(10): 518-21. 
10 Slama K, Redman S, Perkins J, Reid AL, Sanson-Fisher RW. The effectiveness of two smoking cessation programmes for use in general practice: a randomised clinical trial. British Medical Journal 1990; 300(6741): 1707-9.

11 Wallace P, Cutler S, Haines A. Randomised controlled trial of general practitioner intervention in patients with excessive alcohol consumption. British Medical Journal 1988; 297(6649): 663-8.

12 Elley CR, Kerse N, Arroll B, Robinson E. Effectiveness of counselling patients on physical activity in general practice: cluster randomised controlled trial. British Medical Journal 2003; 326(7393): 793.

13 Campbell MJ, Browne D, Waters WE. Can general practitioners influence exercise habits? Controlled trial. British Medical Journal (Clinical Research Edition) 1985; 290(6474): 1044-6.

14 Colvin RH, Olson SB. A descriptive analysis of men and women who have lost significant weight and are highly successful at maintaining the loss. Addictive Behaviors 1983; 8(3): $287-95$.

15 Laws R. A new evidence-based model for weight management in primary care: The Counterweight Programme. Journal of Human Nutrition and Dietetics 2004; 17(3): 191-208.

16 Huang N, Naccarella L. Active Script: Stepping Ahead in Year 2. Melbourne: Victoria Council on Fitness and General Health Incorporated, October, 2001.

17 Lawlor DA, Keen S, Neal RD. Can general practitioners influence the nation's health through a population approach to provision of lifestyle advice? British Journal of General Practice 2000; 50(455): 455-9.

18 Laws R. Current approaches to obesity management in UK primary care: The Counterweight Programme. Journal of Human Nutrition and Dietetics 2004; 17(3): 183-90.

19 Kristeller JL, Hoerr RA. Physician attitudes toward managing obesity: differences among six specialty groups. Preventive Medicine 1997; 26(4): 542-9.

20 Silagy C, Muir J, Coulter A, Thorogood M, Yudkin P, Roe L. Lifestyle advice in general practice: rates recalled by patients. British Medical Journal 1992; 305(6858): 871-4.

21 O'Brien SH, Holubkov R, Reis EC. Identification, evaluation, and management of obesity in an academic primary care center. Pediatrics 2004; 114(2): e154-9.

22 Demerath E, Muratova V, Spangler E, Li J, Minor VE, Neal WA. School-based obesity screening in rural Appalachia. Preventive Medicine 2003; 37(6): 553-60.
23 Heath C, Grant W, Marcheni P, Kamps C. Do family physicians treat obese patients? Family Medicine 1993; 25(6): 401-2.

24 Stafford RS, Farhat JH, Misra B, Schoenfeld DA. National patterns of physician activities related to obesity management. Archives of Family Medicine 2000; 9(7): 631-8.

25 Beresford SA, Curry SJ, Kristal AR, Lazovich D, Feng Z, Wagner $\mathrm{EH}$. A dietary intervention in primary care practice: The Eating Patterns Study. American Journal of Public Health 1997; 87(4): 610-6.

26 van Binsbergen JJ, Drenthen AJ. Patient information letters on nutrition: development and implementation. American Journal of Clinical Nutrition 2003; 77(4 Suppl.): S1035-8.

27 Morris SE, Lean ME, Hankey CR, Hunter C. Who gets what treatment for obesity? A survey of GPs in Scotland. European Journal of Clinical Nutrition 1999; 53(2 Suppl.): S44-8.

28 Kushner RF. Barriers to providing nutrition counseling by physicians: a survey of primary care practitioners. Preventive Medicine 1995; 24(6): 546-52.

29 Fogelman Y, Vinker S, Lachter J, Biderman A, Itzhak B, Kitai E. Managing obesity: a survey of attitudes and practices among Israeli primary care physicians. International Journal of Obesity and Related Metabolic Disorders 2002; 26(10): 1393-7.

30 Paxton S, Tighe C. Weight loss referral practices and recommendations of general practitioners. Australian Journal of Nutrition and Dietetics 1994; 51(2): 82-7.

31 Moore H, Summerbell CD, Greenwood DC, Tovey P, Griffiths J, Henderson $\mathrm{M}$, et al. Improving management of obesity in primary care: cluster randomised trial. British Medical Journal 2003; 327(7423): 1085.

32 Sayer G, Britt H, Horn F. Measure of Health and Health Care Delivery in General Practice in Australia. Canberra: Australian Institute of Health and Welfare, 2000.

33 Steptoe A, Doherty S, Kendrick T, Rink E, Hilton S. Attitudes to cardiovascular health promotion among GPs and practice nurses. Family Practice 1999; 16(2): 158-63.

34 Lazarus K. Nutrition practices of family physicians after education by a physician nutrition specialist. American Journal of Clinical Nutrition 1997; 65(6 Suppl.): S2007-9.

35 Maiburg BH, Rethans JJ, Schuwirth LW, Mathus-Vliegen LM, van Ree JW. Controlled trial of effect of computer-based nutrition course on knowledge and practice of general practitioner trainees. American Journal of Clinical Nutrition 2003; 77(4 Suppl.): S1019-24. 